\title{
Modelado de la Transferencia de Calor en el Tratamiento Térmico de Productos Enlatados
}

\author{
Fernando A. López-Mata(1), José J. Valencia-López ${ }^{(1)}$ y Luis Medina-Torres ${ }^{(2)}$ \\ (1) Departamento de Procesos y Tecnología, División de Ciencias Naturales e Ingeniería, Universidad \\ Autónoma Metropolitana Unidad Cuajimalpa, Av. Vasco de Quiroga 4871, Cuajimalpa de Morelos, Santa Fe \\ Cuajimalpa, 05348 Ciudad de México, México. \\ (2) Facultad de Química, Universidad Nacional Autónoma de México, Ciudad de México, México \\ (e-mail: jvalencia@correo.cua.uam.mx)
}

Recibido Dic. 11, 2015; Aceptado Feb. 16, 2016; Versión final Abr. 16, 2016, Publicado Dic. 2016

\begin{abstract}
Resumen
Se presenta un modelo matemático y su solución numérica en un software de Dinámica de Fluidos Computacional (CFD, por sus siglas en inglés) para la determinación de la transferencia de calor sobre un producto alimenticio enlatado con propiedades reológicas no-Newtonianas. Se analiza la transferencia de calor dentro de la lata con el fin de obtener perfiles de temperatura que ayuden a determinar las condiciones óptimas de transferencia de calor en el proceso de esterilización de productos enlatados. De igual manera se analiza la influencia de la transferencia de calor en la cinética de destrucción de microorganismos patógenos como la bacteria productora de esporas Clostridium botulinum.
\end{abstract}

Palabras clave: dinámica de fluidos computacional; transferencia de calor; producto enlatado; leche condensada

\section{Modeling of Heat Transfer in the Thermal Treatment of Canned Products}

\begin{abstract}
A mathematical model and its numerical solution in Computational Fluid Dynamics software (CFD, for its acronym in English) for the determination of heat transfer on a foodstuff canned, with non-Newtonian rheological properties. The heat transfer inside the can is analyzed in order to obtain temperature profiles that help to determine the optimum conditions of heat transfer in the process of sterilization of canned products. In addition, the influence of heat transfer on the kinetics of destruction of pathogenic microorganisms such as the spore-producing bacteria Clostridium botulinum, is analyzed.
\end{abstract}

Keywords: computational fluid dynamics; heat transfer; canned product; condensed milk 


\section{INTRODUCCIÓN}

En la actualidad los tratamientos térmicos siguen siendo la manera más utilizada de preservar los alimentos, desde que fue propuesta por Nicolás Appert en 1810, independientemente del desarrollo de tecnologías alternativas denominadas tratamientos no térmicos (campo de pulsos eléctricos, alta presión hidrostática, UV, entre otros) (Farid y Ghani, 2002). Existen dos métodos diferentes de procesamiento térmicos convencionales aplicados en la industria alimentaria: el procesamiento aséptico, en donde el producto alimenticio es esterilizado antes de ser envasado y aquel en el que el producto alimenticio se envasa y después se esteriliza (Erdogdu et al., 2010). Siendo uno de los procesos industriales de mayor consumo energético e inversión de capital representando entre el 10 y $15 \%$ de las manufacturas totales (Dincer, 1998), razones por las que continúan abiertas líneas de investigación enfocadas a la mejora continua de los procesos térmicos con la finalidad de reducir costos de producción, optimizar el uso de recursos energéticos y maximizar la retención de nutrientes. El objetivo final es procesar alimentos seguros, de alta calidad y a un precio que el consumidor esté dispuesto a erogar (Ghani, 2006).

El diseño de un proceso térmico, requiere la identificación del microorganismo a inactivar y que guarda relación con el producto alimenticio. Para alimentos de baja acidez $(\mathrm{pH}>4.6)$ como es el caso de la leche condensada, se da una atención especial al Clostridium botulinum, microorganismo que se toma como base para el diseño de procesos de esterilización, ya que una bacteria anaerobia formadora de esporas resistentes al calor y productor de una toxina letal para el hombre aún en concentraciones de $10 \mathrm{ng} / \mathrm{kg}$ de peso corporal (Alvarado et al., 2009). El tratamiento térmico de alimentos no es selectiva por lo que son inherentes al proceso pérdidas del valor nutricional y sensorial del alimento. El diseño efectivo de un proceso térmico, requiere del conocimiento de cinéticas de destrucción de microorganismos, enzimas y nutrientes asociados a la calidad del alimento, además de perfiles de temperatura de la zona de calentamiento más lento (ZCL) del envase (Alvarado et al., 2009).

Debido a la cinética de primer orden que sigue la inactivación microbiana es prácticamente imposible la obtención de un producto estéril. La destrucción completa de formas viables, representaría la destrucción del alimento (Valle y Merson, 1981). Los tratamientos térmicos, además de inactivar microorganismos, también destruyen algunas vitaminas termolábiles como la tiamina y promueven la oxidación de lípidos ocasionando un sabor desagradable al producto alimenticio. Los efectos del calor sobre proteínas y lípidos han sido descritos con detalle por Mauron en 1982, Witting y Dimick en 1982 (Fellows, 2000). Motivo por el cual la industria alimenticia se ha dado a la tarea de investigar mejoras en la tecnología del procesamiento térmico como la agitación (Singh y Ramaswamy, 2015) o el procesamiento aséptico con el fin de proporcionar una buena calidad del alimento sin comprometer la seguridad de la salud pública (Singh et al., 2015).

Para describir la cinética de inactivación térmica de microorganismos productores de esporas es necesario conocer dos parámetros: el tiempo de reducción decimal (D) y el valor $\mathrm{Z}_{\mathrm{T} . \text {.. El tiempo de reducción decimal }}$ $(D)$ es el tiempo de procesamiento aplicado a una población microbiana a temperatura constante, requerido para inactivar el $90 \%$ de la población con cinética de muerte de primer orden. Para $C$. botulinum $D=0,21$ min a $121.1^{\circ} \mathrm{C}$ (Alvarado et al., 2009). El valor $Z_{T}$ se expresa como la diferencia de temperatura requerida para un cambio decimal en el valor $\mathrm{D}$, este valor es particular para cada microorganismo a inactivar. Para $C$. botulinum $\mathrm{Z}_{\mathrm{T}}=10^{\circ} \mathrm{C}$ (Alvarado et al., 2009). La ubicación térmica de la ZCL, ha sido tradicionalmente medida usando termopares fijados en diversos puntos dentro del envase. Sin embargo, el uso de termopares incurre en errores de medición asociados a la perturbación de los patrones de flujo y el incremento del área de transferencia de calor, además de que la ZCL no se mantiene fija (Jiménez et al., 2005) y su trayectoria depende de la forma y orientación del envase, las propiedades termodinámicas del alimento y de la dinámica de calentamiento. (Varma y Kannan, 2006).

Los mecanismos más importantes de transmisión de calor en el procesamiento térmico de alimentos son: conducción, convección natural y/o forzada. Otro factor importante son las propiedades termofísicas de los alimentos en los procesos térmicos ya que éstas determinan la velocidad de transferencia de calor en el interior del producto. Así que en los procesos térmicos de calentamiento y enfriamiento se ha establecido que las propiedades primarias comprenden: la conductividad térmica, el calor específico y la densidad del alimento (Ashrae, 1977). Si la transferencia de calor es controlada por conducción, la ZCL se localizará en el centro geométrico del envase, teniendo poco efecto sobre su ubicación, la dependencia de las propiedades termodinámicas con la temperatura (Lucatero, 2000). La convección natural por su parte, provoca que la ZCL migre hacia el fondo del envase durante el proceso térmico, desplazándose hacia el eje axial y luego hacia el centro de la lata (Jiménez et al., 2005).

Los modelos matemáticos proporcionan una de las herramientas básicas para describir procesos físicos y predecir su comportamiento en condiciones variadas. A través de su uso es posible el diseño y mejorar el 
entendimiento de proceso en el área de ingeniería alimentaria. Una simulación del proceso utilizando un modelo matemático es esencial para la optimización y el control de procesos. (Tirado et al., 2007). Asi que debido a la dificultad de ubicar la ZCL para alimentos enlatados y los errores asociados al uso de termopares, han generado interés en el entendimiento de los fenómenos de transporte asociados a dicho proceso, lo que implica la solución numérica de las ecuaciones de conservación de momentum, energía y masa de manera acoplada, convirtiéndolo en un problema de convergencia difícil. La solución no hubiera sido posible, sin la evolución de los sistemas de cómputo de alta velocidad, que permitieron el desarrollo de software de Dinámica de Fluidos Computacional (CFD). La simulación numérica provee información más detallada y precisa sobre la distribución de temperatura y patrones de flujo dentro del envase. Por lo anterior, es de gran importancia plantear un modelo que describa la transferencia de conservación de cantidad de movimiento, masa y energía.

Varios modelos matemáticos predictivos han sido empleados para la estimación de la distribución de temperatura para un alimento enlatado. En cuanto a los alimentos líquidos enlatados muchos investigadores han evaluado experimentalmente el coeficiente de transferencia de calor durante el tratamiento térmico y han registrado el efecto de diferentes procesos y variables de productos dependiendo el coeficiente de transferencia de calor (Singh et al., 2015).

\section{METODOLOGÍA}

Se plantea el problema en función de las leyes de conservación de cantidad de movimiento y energía que constituyen la base de estudio del fenómeno convectivo, las cuales describen las variaciones de velocidad y temperatura con respecto al tiempo y posición en el sistema (Moraga et al., 2011). El problema planteado se resuelve tomando en cuenta una lata individual de radio $R$ y altura $L$ (ver Figura 1), la cual se encuentra a una temperatura inicial To, que contiene un alimento constituido por un fluido homogéneo. Se resuelve un caso axisimétrico que permite modelar un problema originalmente en 3-D como uno en 2-D, debido a la geometría cilíndrica del envase a modelar. Fluido en régimen laminar, no-Newtoniano, isotrópico. El modelo planteado se resuelve y se valida con resultados publicados por (Moraga et al., 2011) una vez validado el modelo, se resuelve el mismo caso de estudio pero con los datos fisicoquímicos y reológicos que describen a la leche condensada.

\section{Planteamiento del modelo}

Se dibuja una geometría rectangular con dimensiones de $0.12 \mathrm{~m}$ de altura y $0.05 \mathrm{~m}$ de radio, se construyó una malla en forma tetraédrica

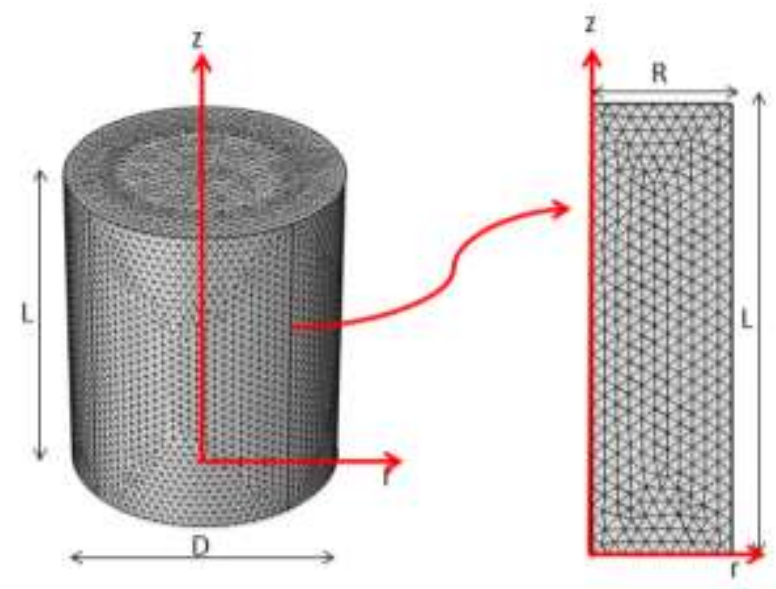

Fig. 1. Geometría considerada para el modelo

\section{Ecuación de continuidad}

$$
\frac{\partial v_{r}}{\partial r}+\frac{v_{r}}{r}+\frac{\partial v_{z}}{\partial z}=0
$$

Ecuación de cantidad de movimiento en la dirección (r)

$$
\bar{\rho}\left[\frac{\partial v_{r}}{\partial t}+v_{r} \frac{\partial v_{r}}{\partial r}+v_{z} \frac{\partial v_{r}}{\partial r}\right]=-\frac{\partial p}{\partial z}+\mu\left[\frac{\partial^{2} v_{r}}{\partial r^{2}}+\frac{1}{r} \frac{\partial v_{r}}{\partial r}+\frac{\partial^{2} v_{r}}{\partial z^{2}}\right]
$$


Ecuación de cantidad de movimiento en la dirección ( $\mathrm{z}$ )

$\bar{\rho}\left[\frac{\partial v_{z}}{\partial t}+v_{r} \frac{\partial v_{z}}{\partial r}+v_{z} \frac{\partial v_{z}}{\partial r}\right]=-\frac{\partial p}{\partial z}+\mu\left[\frac{\partial^{2} v_{z}}{\partial r^{2}}+\frac{1}{r} \frac{\partial v_{z}}{\partial r}+\frac{\partial^{2} v_{z}}{\partial z^{2}}\right]+g \rho_{\text {ref }}[1-\beta(T-$ Tref $)]$

Ecuación de Energía

$\bar{\rho} C p\left[\frac{\partial T}{\partial t}+v_{z} \frac{\partial T}{\partial z}+v_{r} \frac{\partial T}{\partial r}\right]=k\left[\frac{\partial^{2} T}{\partial r^{2}}+\frac{1}{r} \frac{\partial T}{\partial r}+\frac{\partial^{2} T}{\partial z^{2}}\right]$

Modelo de ley de la potencia para la viscosidad

$\tau_{x y}=m\left(-\frac{d v_{y}}{d x}\right)^{n}=m(\dot{\gamma})^{n}$

Condición inicial

$t=0 \mathrm{~s} \quad T=25^{\circ} \mathrm{C}$

Condiciones de Frontera

En $r=0 ; \quad \frac{\partial T}{\partial r}=0 \quad \frac{\partial v_{r}}{\partial r}=0$

En $r=R ; \quad v_{r}=0 \quad v_{z}=0 ; T=121^{\circ} \mathrm{C}$

En $z=0 ; \quad v_{r}=0 \quad v_{z}=0 ; T=121^{\circ} \mathrm{C}$

En $z=L ; \quad v_{r}=0 \quad v_{z}=0 ; T=121^{\circ} \mathrm{C}$

En la Figura 2 se muestra el modelo en 2-D indicando las condiciones de frontera para la geometría construida.

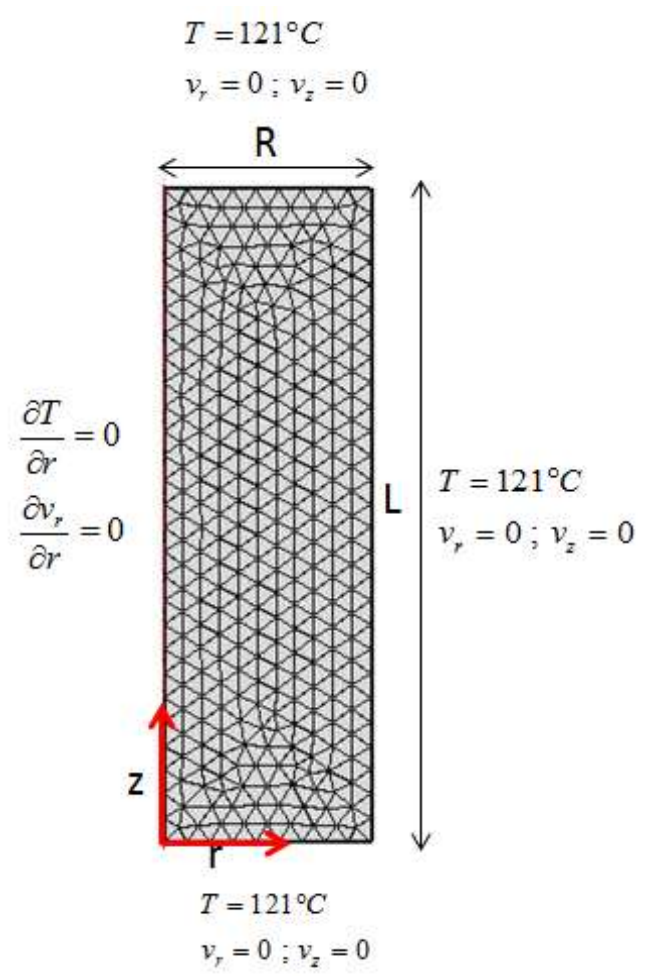

Fig. 2. Modelo en 2-D con condiciones de frontera 
Para la determinación de la inactivación microbiana $(U)$ se utiliza la ecuación propuesta por Bhuvaneswari y Anandharamakrishnan. (Bhuvaneswari et al., 2014)

$$
U=10^{\frac{T-T_{r e f}}{Z_{T}}}
$$

\section{RESULTADOS Y DISCUSIÓN}

Se presentan los resultados en dos etapas: los resultados que validan el modelo y luego una parte donde se muestra un caso de estudio, utilizando el modelo ya validado.

\section{Validación del modelo}

Antes de resolver las ecuaciones para el modelo de la leche condensada, se realiza una validación del modelo comparándolo con los resultados obtenidos en un artículo previo publicado por (Moraga et al., 2011), en el cual se presenta un modelo sobre la transferencia de calor por convección natural durante la esterilización de un fluido no-Newtoniano enlatado, utilizando como fluido modelo Carboximetil Celulosa (CMC), los parámetros fisicoquímicos utilizados se muestran en la Tabla 1.

Tabla 1 Propiedades fisicoquímicas y reológicas fluido modelo

\begin{tabular}{|l|c|c|}
\hline Propiedad & Valor & Unidades \\
\hline Temperatura caliente $(T h)$ & 394 & $\mathrm{~K}$ \\
\hline Temperatura de referencia $\left(T_{\text {ref }}\right)$ & 287 & $\mathrm{~K}$ \\
\hline Densidad $(\rho)$ & 1032 & $\mathrm{Kg} / \mathrm{m}^{3}$ \\
\hline Capacidad calorífica $(C p)$ & 984 & $\mathrm{~J} /(\mathrm{Kg} \mathrm{K})$ \\
\hline Conductividad Térmica $(K)$ & 0.57 & $\mathrm{~W} /(\mathrm{m} \mathrm{K})$ \\
\hline Coeficiente de expansión volumétrica $(\beta)$ & 0.0002 & $1 / \mathrm{K}$ \\
\hline Índice de flujo $(n)$ & 0.94 & - \\
\hline Índice de consistencia viscosa $(m)$ & 1.67 & $\mathrm{mPa} s$ \\
\hline
\end{tabular}

La simulación y los resultados obtenidos en la validación del modelo se presentan a continuación, donde se hace una malla fina en forma tetraédrica con 614 elementos a computar en un tiempo de $1500 \mathrm{~s}$ (Moraga N. et al., 2011) (Ver Figura 3).

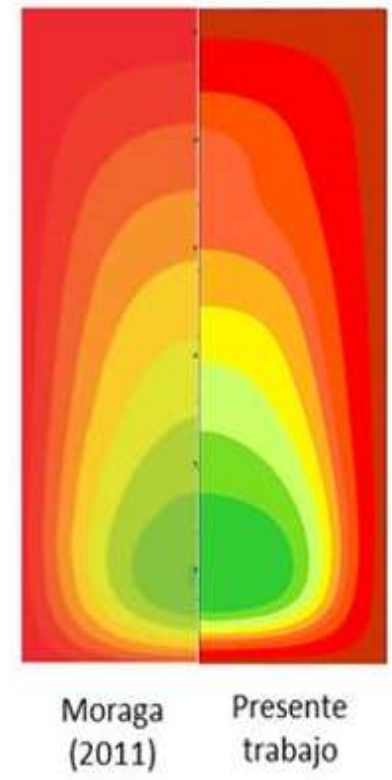

a)

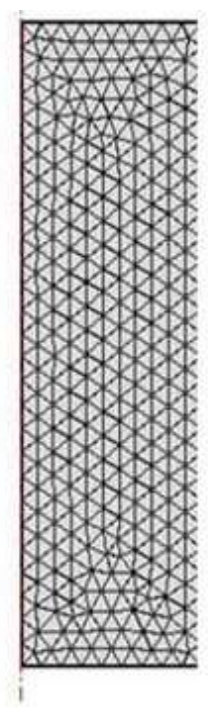

\section{Malla fina con 614 elementos}

b)

Fig. 3 Resultados obtenidos en la validación del modelo con una malla de 614 y 7892 elementos 
En la Figura 3 a) se puede observar que los perfiles de temperatura obtenidos con una malla de 614 elementos son muy similares sin embargo se puede apreciar que existe un perfil en el cual se presenta una perturbación debido al número de elementos resueltos en la malla, es por ello que se hace una nueva simulación tomando los mismos datos de la Tabla 1 pero tomando una malla con 7892 elementos a resolver, obteniendo los resultados que se muestran en la Figura 3 b). En la Figura 3 b) se puede observar que al resolver el modelo una malla extremadamente fina con 7892 elementos con un tiempo de computación de $1000 \mathrm{~s}$ la perturbación que se muestra en la Figura 3 a) en los perfiles de temperatura se corrigió de tal manera que los resultados obtenidos en este trabajo son iguales a los reportados (Moraga et al., 2011).

Es importante señalar que el tipo de malla y el número de nodos a resolver es importante en el modelo, ya que a mayor número de elementos o nodos, los resultados obtenidos serán más confiables aunque el tiempo de resolución será mayor. Una vez validados los perfiles de temperatura y debido a que está provoca un cambio en la densidad y por consiguiente un movimiento del fluido. A continuación se presenta la comparación de las líneas de corriente que describen el movimiento del fluido dentro de la lata para ambos modelos (Ver Fig. 4). En la Figura. 4 se puede observar el movimiento del fluido durante el periodo del tiempo en alcanzar la temperatura de esterilización, donde las líneas de corriente son de tipo oscilatorias posicionándose casi al fondo de la lata debido a las fuerzas de flotación involucradas dentro de la lata.

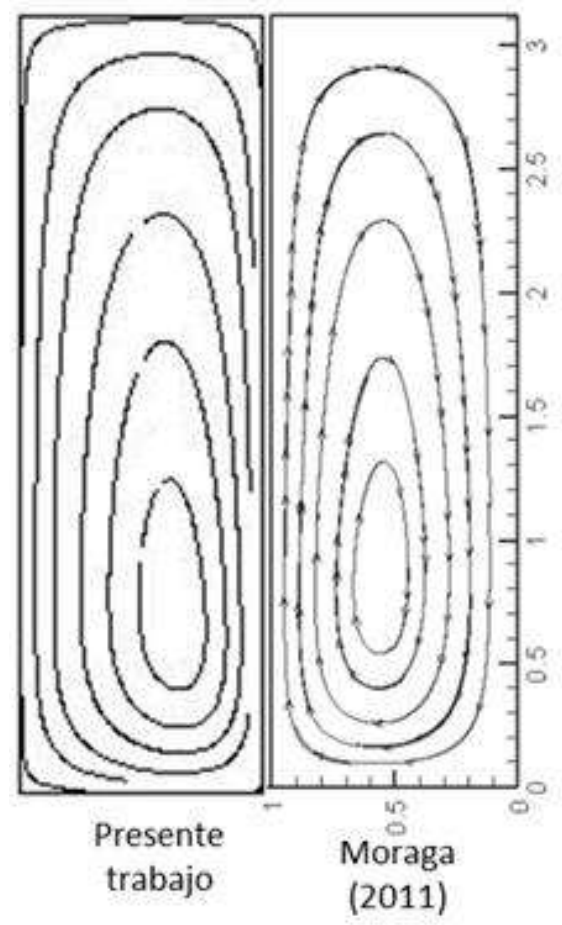

Fig. 4 Líneas de corriente de la distribución del flujo

\section{Caso de estudio: Leche condensada}

Una vez validado el modelo se puede tomar un líquido alimenticio con comportamiento no- Newtoniano para evaluar el tiempo y la temperatura que ayuden a determinar las condiciones óptimas en el proceso de esterilización de productos enlatados. La leche condensada es un tipo de leche evaporada por ser el producto obtenido de la evaporación de agua de la leche entera o leche total o parcialmente descremada con o sin la adición de azúcar. Aunque el producto se suministra a granel como una materia prima para la industria alimentaria la cual se prepara principalmente para el consumo directo donde se empaca en cantidades relativamente pequeñas en latas o en botellas selladas, las cuales se someterán a un proceso térmico. Los parámetros fisicoquímicos y reológicos utilizados para la evaluación del modelo caso de estudio se describen en la Tabla 2.

La simulación y los resultados obtenidos en la validación del modelo se presentan a continuación, donde se realiza un malleo fino en forma tetraédrica con 7892 elementos a computar en un tiempo de $1000 \mathrm{~s}$ (Ver Fig. 5). El periodo de esterilización debe variar de 15 a 20 minutos a temperaturas de $115-116^{\circ} \mathrm{C} 0$ superiores (Van Den Berg, 2001). En la Figura 5 se muestra la formación de los perfiles de temperatura dentro de una lata a tomados en diferente tiempo del proceso de esterilización, resultado de la solución numérica del problema planteado. 
Tabla 2 Parámetros fisicoquímicos y reológicos de la leche condensada.

\begin{tabular}{|l|c|c|}
\hline \multicolumn{1}{|c|}{ Propiedad } & Valor & Unidades \\
\hline Temperatura caliente $(T h)$ & 394 & $\mathrm{~K}$ \\
\hline Temperatura de referencia $\left(T_{\text {ref }}\right)$ & 298 & $\mathrm{~K}$ \\
\hline Densidad $(\rho)$ & 1032 & $\mathrm{Kg} / \mathrm{m}^{3}$ \\
\hline Calor específico $(C p)$ & 984 & $\mathrm{~J} / \mathrm{Kg} / \mathrm{K}$ \\
\hline Conductividad térmica $(k)$ & 0.57 & $\mathrm{~W} / \mathrm{m} / \mathrm{K}$ \\
\hline Coeficiente de expansión volumétrica $(\beta)$ & 0.0002 & $1 / \mathrm{K}$ \\
\hline Índice de flujo $(\mathrm{n})$ & 0.94 & - \\
\hline Índice de consistencia viscosa $(\mathrm{m})$ & 1.67 & $\mathrm{mPa} \mathrm{s}$ \\
\hline
\end{tabular}

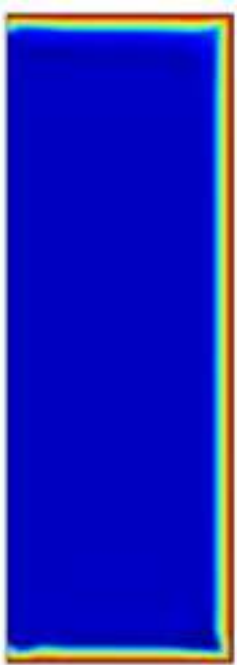

a)

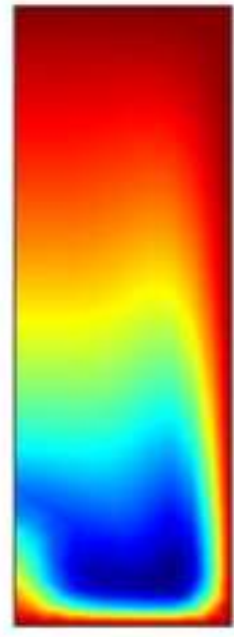

d)

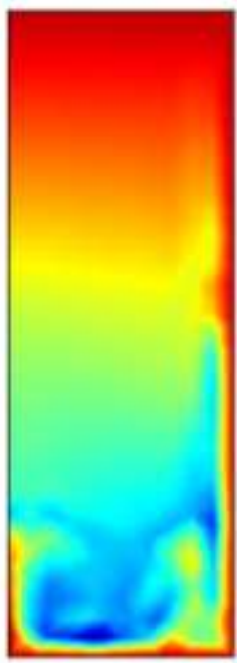

b)

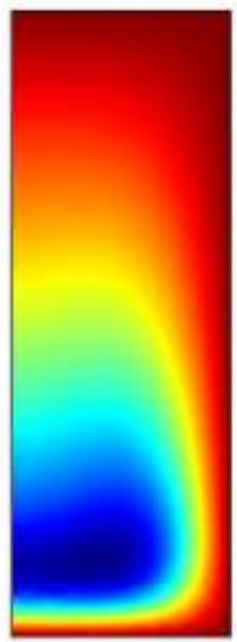

e)

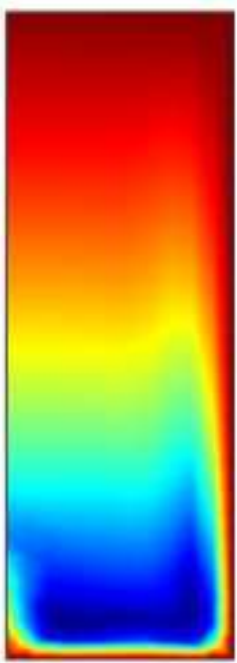

${ }^{\circ} \mathrm{C}$

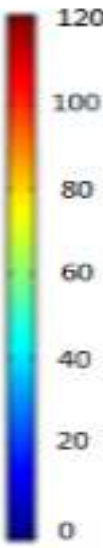

c)
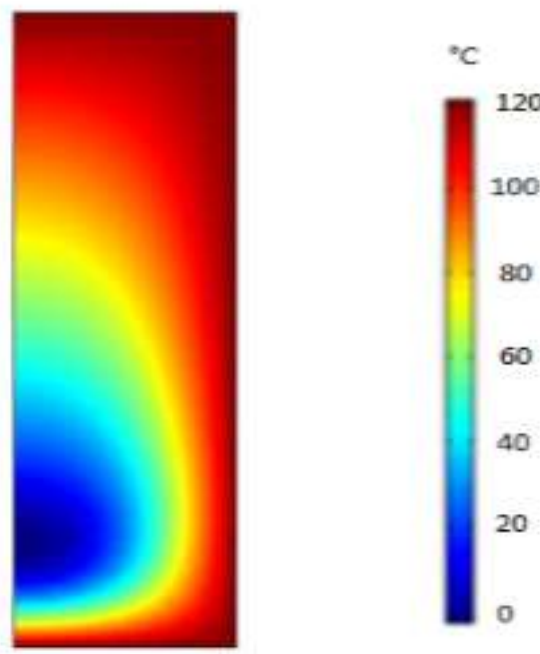

f)

Fig. 5. Capturas de imágenes sobre los perfiles de temperatura dentro de la lata tomadas en diferente tiempo de esterilización, a) $0 \mathrm{~min}$, b) $1 \mathrm{~min}$, c) $3 \mathrm{~min}$, d) $5 \mathrm{~min}$, e) $10 \mathrm{~min}$, f) $15 \mathrm{~min}$

En la Figura 5 se puede observar la formación de los perfiles de temperatura, donde a los 3 minutos del proceso de esterilización se comienzan a formar perfiles tipo herradura característicos de los procesos de tratamiento térmico de productos enlatados (Galotto et al., 2011), cuando el tiempo de esterilización es de 15 minutos se puede observar la clara formación de los perfiles de temperatura de tipo herradura los cuales se obtienen cuando la lata alcanza el estado estacionario $\left(\sim 121^{\circ} \mathrm{C}\right)$, donde la zona de calentamiento más lenta está indicada en color azul la cual se posiciona al fondo de la misma debido a las propiedades del 
fluido y las fuerzas de flotación y gravimétricas que están actuando en el proceso de convección. En el artículo de (Erdogdu et al., 2010) analizan la transferencia de calor de una lata que contiene agua (fluido Newtoniano) en el cual se menciona que el del fluido con mayor temperatura comienza a desplazarse hacia arriba hasta que es desviado por la superficie de la lata generando un movimiento en la dirección radial y el fluido con menor temperatura es desplazado hacia la parte inferior ocasionando una recirculación del flujo y aumentando la transferencia de calor ubicando así la ZCL en el centro de la lata. En la Figura 5 se puede observar que el efecto del movimiento del fluido descrito por (Erdogdu et al., 2010) es similar al movimiento que se presenta en la leche condensada (fluido no-Newtoniano), ya que los perfiles de temperatura de tipo herradura que se forman pueden ser diferentes según sean las variaciones de la densidad del fluido ocasionadas por los gradientes de temperatura que se tienen en los diferentes puntos dentro de la lata.

En la Figura 6 se muestra una gráfica que describe la temperatura de esterilización y la cinética de destrucción de la bacteria Clostridium botulinum en función del tiempo y podemos observar que aunque la temperatura de esterilización llego a un estado estacionario de $121^{\circ} \mathrm{C}$ a los $\sim 20$ minutos, la cinética de inactivación en ese tiempo aún no llega a un estado estacionario, ya que hasta llegar a los 25 minutos existe una variación de bacterias inactivadas. Lo cual indica que aunque la lata tenga una temperatura constante de $121^{\circ} \mathrm{C}$ existe un tiempo en el cual se debe dejar a esa temperatura para lograr un proceso de esterilización deseado. Se considera que un alimento se encuentra estéril cuando la carga microbiana se reduce en un factor de $10^{12}$ (Jiménez, 2005)

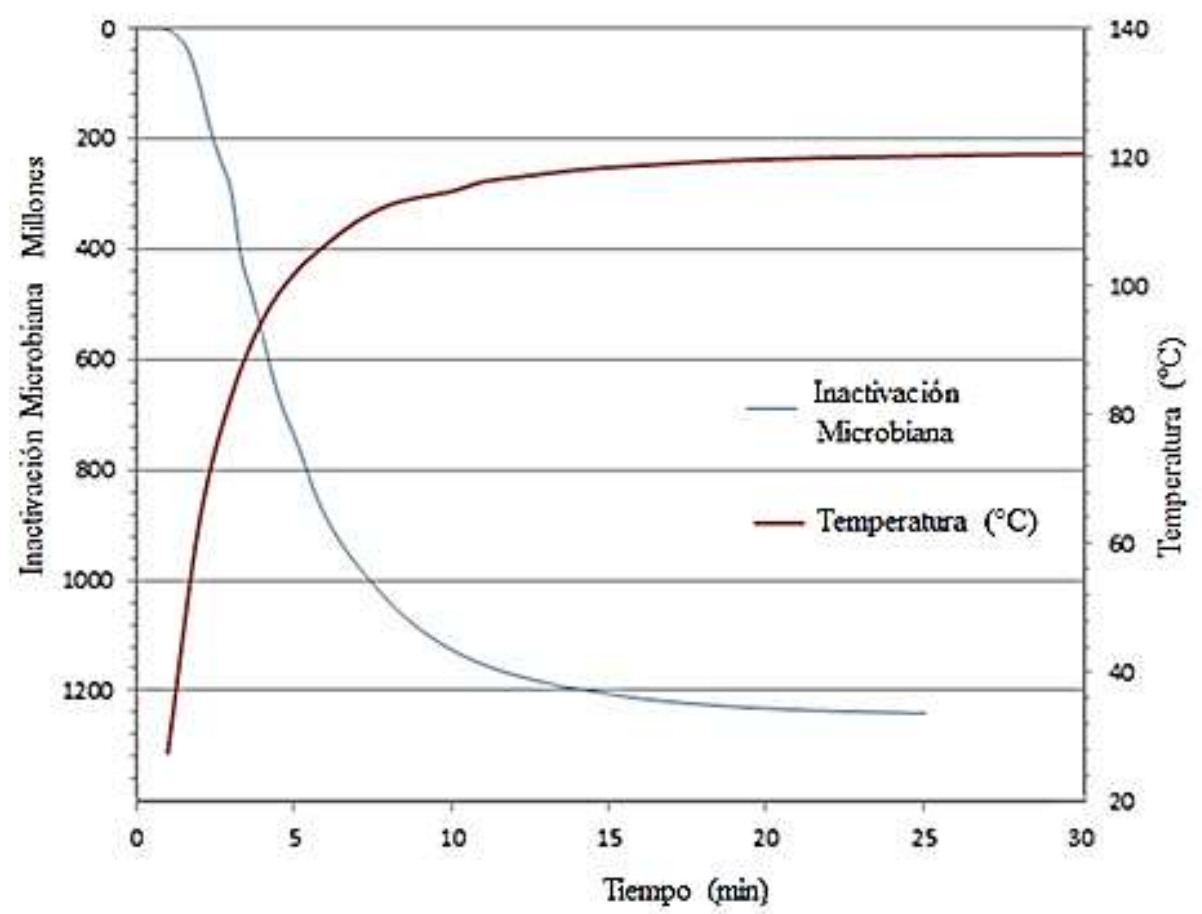

Fig. 6: Gráfica de temperatura de esterilización e inactivación de microorganismos patógenos

El tratamiento térmico de un alimento líquido como es el caso de la leche condensada debe llevarse a cabo después del enlatado para lograr una destrucción mínima de nutrientes y aspectos físicos como color y textura. En este trabajo se planteó un modelo de trasferencia de calor con convección natural libre donde se transfiere el calor de forma natural hacía todo el alimento enlatado debido a las fuerzas de flotación que se originan en el envase tal y como se muestra en la Figura 5. Por otro lado la convección forzada se transfiere el calor por aplicación externa de energía como la agitación giratoria o lateral de la lata con lo cual se mejora la transferencia de calor (Singh et al., 2015).

\section{CONCLUSIONES}

Se cuenta con un modelo en 2D para la transferencia de calor y de cantidad de movimiento para un fluido no-Newtoniano, dicho modelo resuelve las ecuaciones diferenciales parciales de manera simultánea mediante el método de elemento finito con un software comercial de Dinámica de Fluidos Computacional, con el cual se puede estimar: los perfiles de temperatura a través del tiempo, el movimiento del fluido contenido en la lata ocasionado por los efectos de calentamiento convectivo y efectos viscosos, el tiempo que tarda en llegar a la temperatura de esterilización y conocer la cinética de inactivación de microorganismos patógenos. 
Es importante conocer los perfiles de temperatura que gobiernan el proceso de esterilización dentro de la lata ya que con estos es posible conocer la cinética de destrucción de microorganismos patógenos, aunque cabe señalar que dicha cinética presentada en este trabajo fue calculada para una bacteria en especial Clostridium botulinum, esta bacteria es tomada como base para diseñar procesos de esterilización de alimentos enlatados debido a que es una bacteria termo resistente, entonces si se inactiva esta bacteria podemos asegurar que los demás microorganismos patógenos también han sido inactivados y así poder tener un alimento inocuo. Con la solución del modelo plateado en este trabajo es posible observar las condiciones de operación del proceso de esterilización y así poder optimizar el proceso.

\section{REFERENCIAS}

Alvarado J., Botello E., Calderón M., Jiménez H., Martínez G., Navarrete J. Fenomenología de la esterilización de alimentos líquidos enlatados. Revista de la Facultad Ingeniería Universidad de Antioquia: 50, 87-98 (2009)

Ashrae, Fundamentals Handbook. Thermal Properties of Foods, American Society of Heating. Refrigerating and Air Conditioning Engineers Inc. New York, N.Y. USA. (1977)

Bhuvaneswari E., Anandharamakrishnan C. Heat transfer analysis of pasteurization of bottled beer in a tunnel pasteurizer using computational fluid dynamics. Innovative Food Science and Emerging Technologies: 23, 156-163 (2014)

Dincer, I. Heat transfer during heat sterilization and cooling processes of canned products. International Communications of Heat and Mass Transfer: 34, 101-105 (1998)

Erdogdu F., Uyar R., Palazoglu T.K., Experimental comparison of natural convection and conduction heat transfer, doi: 10.1111/j.1745-4530.2008.00309.x, Journal of Food Process Engineering 33, 85-100, (2010)

Fellows, P. J. Food Processing Technology. $2^{\text {nd }}$ Edition, Woodhead Publishing Limited, England (2000)

Tirado F., Acevedo D., Puello P., Determinación Computacional del Coeficiente de Transferencia de Calor y Propiedades Termofísicas de Alimentos, doi: 10.4067/S0718-07642014000300008, Información Tecnológica, 25 (3), 53-58, (2014)

Ghani, A. G. A computer simulation of heating and cooling liquid food during sterilization process using computational fluid dynamics. Association for Computing Machinery New Zealand Bulletin: 3, ISSN 11769998 (2006) (En línea) http://journaldatabase.info/download/pdf/computer_simulation_heating_cooling. (2013)

Ghani, A. G., Farid, M. M. y Chen, X. D. Numerical simulation of transient temperature and velocity profiles in a horizontal can during sterilization using computational fluid dynamics, Journal of Food Engineering: 51, 77-83 (2002)

Hosrt Dieter Tscheuschner, Fundamentos de tecnología de los alimentos, Editorial Zaragoza, España, 300303, (2001)

Jiménez H., González L. M., Botello, J.E., Navarrete J. L., Estudio Numérico de la Esterilización Térmica de Alimentos Líquidos Enlatados que Contienen Partículas, Empleando el Enfoque de Medio Poroso. Revista Mexicana de Ingeniería Química, 4, 1-23 (2005)

Lucatero, C.S. Estudio numérico de la transferencia de calor en la esterilización de alimentos. Tesis de Licenciatura. Instituto Tecnológico de Celaya, Celaya Gto (2000)

Moraga N., Torres A., Galotto M. J., Guarda A., Non-newtonian canned liquid food, unsteady fluid Mechanics and heat transfer prediction for Pasteurization and sterilization. Journal of Food Process Engineering: 34, 2000-2025 (2001)

Singh A., Ramaswamy H., Effect of product related parameters on heat-transfer rates to canned particulate non-Newtonian fluids (CMC) during reciprocation agitation thermal processing. doi: 10.1016/j.jfoodeng.2015.05.004. Journal of Food Engineering: 165, 1-12, (2015)

Singh A., Pratap A., Ramaswamy H., Computational techniques used in heat transfer studies on canned liquid particulate mixtures. doi: 10.1016/j.tifs.2015.02.001 Trends in Food Science \& Technology: 43, 83-103, (2015) 
Valle P., Merson R. L., Cálculo del tiempo de tratamiento térmico en botes, Método General y Gráfico. Tecnología de Alimentos 16 (3), 10-22 (1981)

Varma, M.N. y Kannan, A. CFD studies on natural convective heating of canned food in conical and cylindrical containers. Journal of Food Engineering: 4, 1024-1036 (2006)

Van Den Berg J.C.T., Evaporated and condensed milk, Government dairy an Adviser for milk products, The Hague, Netherlands, (2001) 